\title{
Missglückte Auferstehung
}

Sie gilt als die „Killer-Applikation“, an deren Erfolg sich alle Internet-Dienste messen lassen müssen: die E-Mail. Nach Vorläufern aus den frühen 70er Jahren wurde das Format der „ARPA Network Text Messages" am 21.11.1977 in RFC 733 spezifiziert; im August 1982 folgte die Standardisierung des bis heute eingesetzten "Simple Mail Transfer Protocol“ (SMTP) durch Jon Postel. Schon vor dem Siegeszug des World Wide Web (WWW), dessen Basisstandards HTTP und HTML erst 1995/96 als RFC publiziert wurden, hatten sich E-Mails an Universitäten und in vielen Bereichen der Wirtschaft durchgesetzt. Heute sind sie das mit weitem Abstand wichtigste moderne Kommunikationsmittel in und zwischen Unternehmen. Eine Kinderkrankheit ist allerdings bis heute nicht kuriert: E-Mails sind unverschlüsselte Textnachrichten, die über ungeschützte Internet-Verbindungen übertragen werden. Zwar erblickte bereits vor 22 Jahren der erste E-Mail-Sicherheitsstandard das Licht der Welt: In RFC 989 spezifizierte John Linn im Februar 1987 „Privacy Enhancement for Internet Electronic Mail', einen frühen Vorläufer des S/MIME-Standards.

Obwohl zahlreiche E-Mail-Clients heute S/MIME unterstützen und OpenPGP dank der freien deutschen GnuPG-Software komfortabel genutzt werden kann, sind E-Mails nach wie vor meist elektronische Postkarten. Eine willkommene Gelegenheit, behütend einzugreifen:„Der moderne Staat steht deshalb vor der Aufgabe, im elektronischen Kommunikationsraum für eine Grundversorgung an Verbindlichkeit und Vertraulichkeit zu sorgen", so das BSI in der Fachzeitschrift <kes> (1/2008).,"De-Mail“ soll der Dienst heißen, der zunächst in Friedrichshafen am Bodensee pilotiert und ab 2010 mit Hilfe privatwirtschaftlicher „Bürgerportale" einen Schlussstrich unter ungeschützte E-Mail-Kommunikation in Deutschland ziehen soll. De-Mail soll den Versand signierter und verschlüsselter E-Mails von Bürgerportal zu Bürgerportal erlauben, ohne dass Sender und Empfänger Software installieren oder sich um Signatur- und Verschlüsselungsschlüssel kümmern müssen.

Das Konzept krankt jedoch an mehreren Designfehlern. Beim Empfang einer De-Mail bestätigt das Portal dem Empfänger mit qualifizierter Signatur den Eingang - sie gilt damit als zugestellt, unabhängig davon, ob der Empfänger Einsicht in sein Postfach nimmt. Im Postfach liegen De-Mails zudem unverschlüsselt vor, und wer seine Adresse über den De-Mail-Verzeichnisdienst bekannt gibt, muss jederzeit mit rechtswirksamen Zustellungen von Ämtern rechnen.

Bei genauer Betrachtung entpuppt sich De-Mail als allzu offensichtlicher Versuch, die qualifizierte Signatur nach 12 Jahren Misserfolgsgeschichte noch in den Sattel zu heben - und zugleich einen auch für den Bund (als Eigner der Deutschen Post) unerquicklichen Mangel abzustellen: In wachsendem Maße ersetzt der schnelle und quasi kostenfreie E-Mail-Versand den klassischen Brief. Was liegt da näher, als die „Killer-Applikation" mit einem kostenpflichtigen Zusatzservice auszustatten? Tatsächlich gibt es bei DeMail fast nur Gewinner: Die Behörden, deren Prozesskosten für die Bürgerkommunikation sinken, die Bundesregierung, die der elektronischen Signatur endlich zum Durchbruch verhilft, und die Portalanbieter, die zukünftig De-Mails einzeln abrechen können. Nur einer verliert: Der Anwender. Er soll für einen Kommunikationsdienst zahlen, den er bisher kostenfrei nutzt, bei dem er die Inhalte seiner Nachrichten auf zentralen Bürgerportalen preisgibt und Einschreiben nicht mehr frei Haus erhält, sondern selbst rechtzeitig im Portal abholen muss.

Immerhin haben das nicht alle Parlamentarier übersehen. Am 25.04.2009 wurde der Entwurf des Bürgerportal-Gesetzes der Bundesregierung nach einer ebenso klarsichtigen wie vernichtenden Empfehlung der Ausschüsse des Bundesrats in erster Lesung abgelehnt: So sei z.B. die Pflicht des Empfängers zur regelmäßigen Leerung des Postfaches ein unzumutbarer Grundrechtseingriff. Offenbar gibt es noch Volksvertreter, die nicht vergessen haben, dass der Idealtypus des "modernen Staates" kein Leviathan, sondern eine freiheitliche Ordnung ist.

\section{Dirk Fox}

Korrigendum: Anders als im Editorial 6/2009 behauptet, hat der Bundestag bei der BDSG-Novellierung auch die Geldbußen erhöht. Wir bitten den Fehler zu entschuldigen. Operative Hektik ist weder für Gesetze noch für Editorials eine gute Rahmenbedingung. 